\title{
Solving the Fully Fuzzy Bilevel Linear Programming Problem through Deviation Degree Measures and a Ranking Function Method
}

\author{
Aihong Ren \\ Department of Mathematics, Baoji University of Arts and Sciences, Baoji 721013, China \\ Correspondence should be addressed to Aihong Ren; raih2003@hotmail.com
}

Received 10 December 2015; Accepted 23 March 2016

Academic Editor: David Bigaud

Copyright (c) 2016 Aihong Ren. This is an open access article distributed under the Creative Commons Attribution License, which permits unrestricted use, distribution, and reproduction in any medium, provided the original work is properly cited.

\begin{abstract}
This paper is concerned with a class of fully fuzzy bilevel linear programming problems where all the coefficients and decision variables of both objective functions and the constraints are fuzzy numbers. A new approach based on deviation degree measures and a ranking function method is proposed to solve these problems. We first introduce concepts of the feasible region and the fuzzy optimal solution of a fully fuzzy bilevel linear programming problem. In order to obtain a fuzzy optimal solution of the problem, we apply deviation degree measures to deal with the fuzzy constraints and use a ranking function method of fuzzy numbers to rank the upper and lower level fuzzy objective functions. Then the fully fuzzy bilevel linear programming problem can be transformed into a deterministic bilevel programming problem. Considering the overall balance between improving objective function values and decreasing allowed deviation degrees, the computational procedure for finding a fuzzy optimal solution is proposed. Finally, a numerical example is provided to illustrate the proposed approach. The results indicate that the proposed approach gives a better optimal solution in comparison with the existing method.
\end{abstract}

\section{Introduction}

In the past few decades, the bilevel programming problem has been researched from the theoretical and computational points of view [1-6] and has been successfully applied to a variety of fields, such as transport network design [7, 8], principal-agent problems [9], price control [10], and electricity markets [11, 12]. The bilevel programming problem is a hierarchical optimization problem with two levels. This kind of problem is very difficult to solve due to its nonconvexity and nondifferentiability. Over the decades, the vast majority of research on the bilevel programming problem is under deterministic environments. That is to say, all the coefficients and decision variables involved in the problem are crisp values.

However, in most real-world bilevel decision making problems, particularly in logistics planning or human resource planning, it is very hard to determine the values of the coefficients because of incomplete or imprecise information when establishing these models. In this situation, it is more appropriate to apply fuzzy set theory to handle imprecise data [13]. As a matter of fact, the fuzzy bilevel programming problem in which the coefficients either in the objective functions or in the constraints are represented by fuzzy numbers has received much attention of some researchers. Sakawa et al. [14] first considered the fuzzy bilevel programming problem and proposed a fuzzy programming method to deal with the problem. Zhang and $\mathrm{Lu}$ [15] developed an extended Kuhn-Tucker approach based on the new definition of optimal solution to solve this problem. Subsequently, Zhang and $\mathrm{Lu}$ [16] presented a fuzzy bilevel decision making model for a general logistics planning problem and developed a fuzzy number based $K$ th-best approach to find an optimal solution for the proposed model. Zhang et al. [17] gave three approaches to solve such a problem by applying fuzzy set techniques. Dempe and Starostina [18] formulated the fuzzy bilevel programming problem and described one possible approach for formulating a crisp optimization problem being attached to it. In addition, some of the studies in the direction of solving fuzzy multiobjective 
bilevel optimization problems are [19-22]. However, in all of the above-mentioned works, the decision variables were not fuzzy.

In recent years, the fully fuzzy linear programming in which all the coefficients as well as decision variables are described by fuzzy numbers has been an attractive topic for the researchers. Buckley and Feuring [23] considered this kind of problem and employed an evolutionary algorithm to solve it. Based on the concept of the symmetric triangular fuzzy number, the fully fuzzy linear programming problem was transformed into two corresponding linear programming problems, and a lexicography method was developed to cope with the problem [24]. For the fully fuzzy linear programming problem with equality constraints, Kumar et al. [25] presented a new method to find the fuzzy optimal solution of the problem. However, it was shown that Kumar et al.'s model was not correct by Saberi Najafi and Edalatpanah [26], and a new version was provided in their work. Recently, Fan et al. [27] discussed the generalized fuzzy linear programming problem, investigated the feasibility of fuzzy solutions, and proposed a stepwise interactive algorithm based on the idea of design of experiment to deal with the problem. It should be noted that all these works are considered in the case of one-single-level fully fuzzy linear programming.

In reality, decision variables of a fuzzy bilevel programming problem where all the coefficients are fuzzy numbers are also considered as fuzzy numbers. The aim of this paper is to develop a new method to deal with the fully fuzzy bilevel linear programming problem in which all the coefficients and decision variables involved in the problem are expressed as fuzzy numbers. To the best of our knowledge, so far very little information from the existing literature is available on this topic. More recently, Safaei and Saraj [28] proposed a bound and decomposition method to handle the fully fuzzy bilevel linear programming problem. In their approach, the original problem was decomposed into three crisp bilevel linear programming problems in which all of the results obtained by one model are used as constraints' bounds for another model. Sometimes it is difficult to solve the decomposed models separately for the decision makers.

The main contributions of this study are as follows. A new approach based on the deviation degree measures and a ranking function is proposed to find a fuzzy optimal solution of the fully fuzzy bilevel linear programming problem. In order to do so, the feasible region of the fully fuzzy bilevel linear programming problem and the optimality for the upper and lower level objective functions are first discussed, and then the concept of the fuzzy optimal solution is introduced. Second, we apply the deviation degree measures and a ranking function to convert the fully fuzzy bilevel linear programming problem into a deterministic one. In consideration of a balance between improving the upper level objective function value and decreasing the allowed deviation degree for the decision makers, a computational method to derive a fuzzy optimal solution of the fully fuzzy bilevel linear programming problem is presented. Finally, an illustrative numerical example is provided to demonstrate the feasibility of the proposed method. In addition, in comparison with the existing method, the proposed approach gives a better optimal solution.

The remainder of this paper is organized as follows. In Section 2 some basic definitions and preliminary results related to triangular fuzzy numbers are reviewed. In Section 3, we introduce the fully fuzzy bilevel linear programming problem and give a definition of the fuzzy optimal solution of the problem. Then a new method based on the deviation degree measures and a ranking function is proposed to solve the problem. In addition, a computational method to obtain a fuzzy optimal solution of the problem is presented. Section 4 provides a numerical example to illustrate the proposed method. Besides comparisons with the existing method are made to demonstrate the performance of the proposed approach. Finally, Section 5 presents the concluding remarks and suggests directions for future works.

\section{Preliminaries}

In this section, some important concepts and results used in the rest of this paper are introduced.

2.1. Triangular Fuzzy Numbers and the Arithmetic Operations. We first recall the definition of the triangular fuzzy numbers and the algebraic operations.

Let $R$ denote the set of all real numbers.

Definition 1 (see [29]). A fuzzy set $\tilde{a}$ on $R$ is called a triangular fuzzy number if its membership function is described as follows:

$$
\mu_{\tilde{a}}(x)= \begin{cases}\frac{x-a^{(1)}}{a^{(2)}-a^{(1)}}, & a^{(1)} \leq x \leq a^{(2)}, \\ \frac{x-a^{(3)}}{a^{(2)}-a^{(3)}}, & a^{(2)} \leq x \leq a^{(3)}, \\ 0, & \text { otherwise, }\end{cases}
$$

where $a^{(1)}, a^{(3)}$ are the lower bound and the upper bound of $\widetilde{a}$ and $a^{(2)}$ is the most possible value of $\widetilde{a}$, respectively.

The triangular fuzzy number $\tilde{a}$ can be denoted by $\widetilde{a}=$ $\left(a^{(1)}, a^{(2)}, a^{(3)}\right)$. The set of all these triangular fuzzy numbers is denoted by $F(R)$.

Definition 2 (see [29]). A triangular fuzzy number $\widetilde{a}=$ $\left(a^{(1)}, a^{(2)}, a^{(3)}\right)$ is said to be a nonnegative triangular fuzzy number if and only if $a^{(1)} \geq 0$.

Let $\widetilde{a}=\left(a^{(1)}, a^{(2)}, a^{(3)}\right)$ and $\widetilde{b}=\left(b^{(1)}, b^{(2)}, b^{(3)}\right)$ be two triangular fuzzy numbers. The algebraic operations between any two triangular fuzzy numbers $\widetilde{a}$ and $\widetilde{b}$ can be defined by the extension principle [13] and can be expressed as follows:

(i) $\tilde{a} \oplus \widetilde{b}=\left(a^{(1)}+b^{(1)}, a^{(2)}+b^{(2)}, a^{(3)}+b^{(3)}\right)$;

(ii) $\tilde{a} \ominus \widetilde{b}=\left(a^{(1)}-b^{(3)}, a^{(2)}-b^{(2)}, a^{(3)}-b^{(1)}\right)$; 
(iii)

$$
k \widetilde{a}= \begin{cases}\left(k a^{(1)}, k a^{(2)}, k a^{(3)}\right), & k \geq 0, \\ \left(k a^{(3)}, k a^{(2)}, k a^{(1)}\right), & k<0 ;\end{cases}
$$

(iv) let $\widetilde{a}=\left(a^{(1)}, a^{(2)}, a^{(3)}\right)$ be an arbitrary triangular fuzzy number and let $\widetilde{b}=\left(b^{(1)}, b^{(2)}, b^{(3)}\right)$ be a nonnegative triangular fuzzy number; then

$\tilde{a} \otimes \widetilde{b}$

$$
= \begin{cases}\left(a^{(1)} b^{(1)}, a^{(2)} b^{(2)}, a^{(3)} b^{(3)}\right), & a^{(1)} \geq 0, \\ \left(a^{(1)} b^{(3)}, a^{(2)} b^{(2)}, a^{(3)} b^{(3)}\right), & a^{(1)} \leq 0, a^{(3)} \geq 0, \\ \left(a^{(1)} b^{(3)}, a^{(2)} b^{(2)}, a^{(3)} b^{(1)}\right), & a^{(3)}<0 .\end{cases}
$$

In a fuzzy environment, ranking fuzzy numbers plays a very important role in decision making. A ranking function is a function which maps each fuzzy number into the real line, where a natural order exists. Here we use the concepts of ranking fuzzy numbers proposed by Liou and Wang [30].

Definition 3 (see [30]). Let $\tilde{a}=\left(a^{(1)}, a^{(2)}, a^{(3)}\right)$ be a triangular fuzzy number. The ranking function based on expected value of the triangular fuzzy number $\widetilde{a}$ can be defined as $\mathfrak{R}(\widetilde{a})=$ $\left(a_{1}+2 a_{2}+a_{3}\right) / 4$.

2.2. Deviation Degree of Triangular Fuzzy Numbers. Next, we introduce the concept of deviation distances and give the definition of deviation degrees of two triangular fuzzy numbers used in [31,32].

Definition 4 (see [31]). Let $\widetilde{a}=\left(a^{(1)}, a^{(2)}, a^{(3)}\right)$ and $\tilde{b}=$ $\left(b^{(1)}, b^{(2)}, b^{(3)}\right)$ be two triangular fuzzy numbers. The distance between $\tilde{a}$ and $\tilde{b}$ is defined as

$$
d_{p}(\tilde{a}, \tilde{b})=\left(\sum_{h=1}^{3} \alpha^{(h)}\left(\left|a^{(h)}-b^{(h)}\right|\right)^{p}\right)^{1 / p},
$$

where $\alpha^{(1)}=1, \alpha^{(2)}=2$, and $\alpha^{(3)}=1$. $b^{(h)} \mid$.

When $p$ is taken as 1 , we have $d_{1}(\widetilde{a}, \widetilde{b})=\sum_{h=1}^{3} \alpha^{(h)} \mid a^{(h)}-$

Now three kinds of deviation distances of triangular fuzzy numbers can be defined as follows.

Definition 5 (see [32]). Let $\tilde{a}=\left(a^{(1)}, a^{(2)}, a^{(3)}\right)$ and $\tilde{b}=$ $\left(b^{(1)}, b^{(2)}, b^{(3)}\right)$ be two triangular fuzzy numbers; then

(1) the deviation distance of $\tilde{a}$ from $\widetilde{b}$ is

$$
d_{\widetilde{b}}(\widetilde{a})=\sum_{h=1}^{3} \alpha^{(h)}\left|a^{(h)}-b^{(h)}\right| ;
$$

(2) the positive deviation distance of $\widetilde{a}$ from $\widetilde{b}$ is

$$
d_{\widetilde{b}}\left(\tilde{a}^{+}\right)=\sum_{h=1}^{3} \alpha^{(h)} \max \left(a^{(h)}-b^{(h)}, 0\right) ;
$$

(3) the negative deviation distance of $\widetilde{a}$ from $\widetilde{b}$ is

$$
d_{\widetilde{b}}\left(\widetilde{a}^{-}\right)=-\sum_{h=1}^{3} \alpha^{(h)} \min \left(a^{(h)}-b^{(h)}, 0\right) .
$$

The following definition gives the concept of deviation degrees of two triangular fuzzy numbers which normalizes all the deviation distances to the same scale.

Definition 6 (see [32]). Let $\tilde{a}=\left(a^{(1)}, a^{(2)}, a^{(3)}\right)$ and $\tilde{b}=$ $\left(b^{(1)}, b^{(2)}, b^{(3)}\right)$ be two triangular fuzzy numbers; then

(1) the deviation degree of $\widetilde{a}$ from $\widetilde{b}$ is

$$
D_{\widetilde{b}}(\widetilde{a})=\frac{d_{\widetilde{b}}(\widetilde{a})}{\sum_{h=1}^{3} \alpha^{(h)} b^{(h)}}=\frac{\sum_{h=1}^{3} \alpha^{(h)}\left|a^{(h)}-b^{(h)}\right|}{\sum_{h=1}^{3} \alpha^{(h)} b^{(h)}} ;
$$

(2) the positive deviation degree of $\widetilde{a}$ from $\widetilde{b}$ is

$$
\begin{aligned}
D_{\widetilde{b}}\left(\widetilde{a}^{+}\right) & =\frac{d_{\widetilde{b}}\left(\widetilde{a}^{+}\right)}{\sum_{h=1}^{3} \alpha^{(h)} b^{(h)}} \\
& =\frac{\sum_{h=1}^{3} \alpha^{(h)} \max \left(a^{(h)}-b^{(h)}, 0\right)}{\sum_{h=1}^{3} \alpha^{(h)} b^{(h)}} ;
\end{aligned}
$$

(3) the negative deviation degree of $\widetilde{a}$ from $\widetilde{b}$ is

$$
\begin{aligned}
D_{\tilde{b}}\left(\widetilde{a}^{-}\right) & =\frac{d_{\tilde{b}}\left(\widetilde{a}^{-}\right)}{\sum_{h=1}^{3} \alpha^{(h)} b^{(h)}} \\
& =\frac{-\sum_{h=1}^{3} \alpha^{(h)} \min \left(a^{(h)}-b^{(h)}, 0\right)}{\sum_{h=1}^{3} \alpha^{(h)} b^{(h)}} .
\end{aligned}
$$

\section{Methodology}

3.1. Fully Fuzzy Bilevel Linear Programming Problem. Let us consider the following fully fuzzy bilevel linear programming problem in which all the coefficients and the decision variables are fuzzy numbers:

$$
\begin{array}{ll}
\max _{\widetilde{x}_{1}} & \widetilde{c}_{11} \otimes \widetilde{x}_{1} \oplus \widetilde{c}_{12} \otimes \widetilde{x}_{2} \\
& \text { where } \widetilde{x}_{2} \text { solves } \\
\max _{\widetilde{x}_{2}} & \widetilde{c}_{21} \otimes \widetilde{x}_{1} \oplus \widetilde{c}_{22} \otimes \widetilde{x}_{2} \\
\text { s.t. } & \widetilde{a}_{i 1} \otimes \widetilde{x}_{1} \oplus \widetilde{a}_{i 2} \otimes \widetilde{x}_{2} \leq \widetilde{b}_{i}, \quad i=1,2, \ldots, m,
\end{array}
$$

where $\tilde{x}_{1}=\left(\tilde{x}_{11}, \widetilde{x}_{12}, \ldots, \tilde{x}_{1 n_{1}}\right)$ is an $n_{1}$-dimensional fuzzy decision vector controlled by the upper level decision maker and $\widetilde{x}_{2}=\left(\widetilde{x}_{21}, \widetilde{x}_{22}, \ldots, \widetilde{x}_{2 n_{2}}\right)$ is an $n_{2}$-dimensional fuzzy decision vector by the lower level decision maker, elements $\tilde{x}_{j k}$, $j=1,2, k=1,2, \ldots, n_{j}$ of decision vectors $\tilde{x}_{j}$ are nonnegative triangular fuzzy numbers, and $\widetilde{c}_{l j}=\left(\widetilde{c}_{l j 1}, \widetilde{c}_{l j 2}, \ldots, \widetilde{c}_{l j n}\right), l=$ $1,2, j=1,2, \widetilde{a}_{i j}=\left(\widetilde{a}_{i j 1}, \widetilde{a}_{i j 2}, \ldots, \widetilde{a}_{i j n_{j}}\right), i=1,2, \ldots, m$, 
are $n_{j}$-dimensional fuzzy vectors, elements $\widetilde{c}_{l j s}, \tilde{a}_{i j t}, s, t=$ $1,2, \ldots, n_{j}$, of coefficient vectors $\widetilde{c}_{l j}, \tilde{a}_{i j}$, and $\tilde{b}_{i}$ are triangular fuzzy numbers.

Let $F\left(R^{n_{1}}\right)$ and $F\left(R^{n_{2}}\right)$ be the sets of fuzzy vectors over $R^{n_{1}}$ and $R^{n_{2}}$, respectively.

Let us denote by $S=\left\{\left(\tilde{x}_{1}, \tilde{x}_{2}\right) \in F\left(R^{n_{1}}\right) \times F\left(R^{n_{2}}\right) \mid \tilde{a}_{i 1} \otimes\right.$ $\left.\tilde{x}_{1} \oplus \widetilde{a}_{i 2} \otimes \widetilde{x}_{2} \leq \widetilde{b}_{i}, i=1,2, \ldots, m\right\}$, the constraint region of problem (11).

It is clear that problem (11) is an ill-defined problem due to fuzziness of all the coefficients and the decision variables involved in problem (11). That is to say, solution concepts and existing approaches of deterministic bilevel cases cannot be blindly applied to deal with this kind of problem. Therefore, it is required to give proper concepts of the optimal solution and develop potential solution approaches to handle problem (11).

3.2. Definition of the Fuzzy Optimal Solution. In order to solve problem (11), two key questions raised by fuzzy coefficients and fuzzy decision variables involved in the problem should be answered: (1) how to define the feasible region of the problem; (2) how to define the optimality for the upper and lower level objective functions.

First, we discuss the feasible region of problem (11).

For a given $\tilde{x}_{1} \in F\left(R^{n_{1}}\right)$, the lower level decision maker's task is to deal with the problem

$$
\begin{array}{ll}
\max _{\widetilde{x}_{2}} & \widetilde{c}_{21} \otimes \tilde{x}_{1} \oplus \tilde{c}_{22} \otimes \tilde{x}_{2} \\
\text { s.t. } & \tilde{a}_{i 2} \otimes \tilde{x}_{2} \leq \widetilde{b}_{i} \ominus \tilde{a}_{i 1} \otimes \tilde{x}_{1}, \quad i=1,2, \ldots, m,
\end{array}
$$

where each element of the decision vector $\tilde{x}_{2}$ is a nonnegative triangular fuzzy number.

For a given $\widetilde{x}_{1} \in F\left(R^{n_{1}}\right)$, denote the feasible region of the lower level problem (12) by the set

$$
\begin{aligned}
& S\left(\tilde{x}_{1}\right)=\left\{\tilde{x}_{2} \in F\left(R^{n_{2}}\right) \mid \tilde{a}_{i 2} \otimes \tilde{x}_{2} \leq \tilde{b}_{i} \ominus \tilde{a}_{i 1} \otimes \tilde{x}_{1}, i\right. \\
& \quad=1,2, \ldots, m\} .
\end{aligned}
$$

It is worth mentioning that problem (12) is one-singlelevel fully fuzzy linear programming problem with inequality constraints. For this class of problems, it has been pointed out by Hashemi et al. [33] that searching fuzzy optimal solutions is more impressive than obtaining crisp solutions in a fully fuzzy environment. In fact, fuzzy optimal solutions provide ranges of flexibility to the decision makers. However, it is a very difficult task to find the optimal solutions of a fully fuzzy linear programming problem [23].

In this work, we apply a ranking function of fuzzy numbers to define the fuzzy optimal solutions of the lower level problem (12) by Definition 3.

Definition 7. For a given $\tilde{x}_{1} \in F\left(R^{n_{1}}\right)$, a fuzzy vector $\tilde{x}_{2}^{*} \epsilon$ $S\left(\widetilde{x}_{1}\right)$ is said to be a fuzzy optimal solution to the lower level problem (12) if $\mathfrak{R}\left(\widetilde{c}_{21} \otimes \widetilde{x}_{1} \oplus \widetilde{c}_{22} \otimes \widetilde{x}_{2}^{*}\right) \geq \mathfrak{R}\left(\widetilde{c}_{21} \otimes \widetilde{x}_{1} \oplus \widetilde{c}_{22} \otimes \widetilde{x}_{2}\right)$ for any $\tilde{x}_{2} \in S\left(\tilde{x}_{1}\right)$.

Let us denote by $M\left(\tilde{x}_{1}\right)$ the set of fuzzy optimal solutions of the lower level problem (12).
Then the feasible region of the fully fuzzy bilevel optimization problem (11) can be defined as

$$
\operatorname{IR}=\left\{\left(\tilde{x}_{1}, \tilde{x}_{2}\right) \mid\left(\tilde{x}_{1}, \tilde{x}_{2}\right) \in S, \tilde{x}_{2} \in M\left(\tilde{x}_{1}\right)\right\} .
$$

Any $\left(\tilde{x}_{1}, \tilde{x}_{2}\right) \in \operatorname{IR}$ is said to be a bilevel feasible solution.

Obviously, the aim of the upper level decision maker is to maximize the upper level objective function over its feasible space IR.

As we all know that the main complication even in a crisp bilevel programming arises when the solution of the lower level problem is not unique. To treat such an unpleasant situation, the optimistic and the pessimistic strategies are available for the upper level decision maker [2]. We will consider here the optimistic formulation of a fully fuzzy bilevel programming problem. In this way, the upper level decision maker supposes that the lower level decision maker selects the best solution to the upper level decision maker. Then the optimistic formulation of problem (11) is equivalent to the following problem:

$$
\begin{array}{ll}
\max _{\widetilde{x}_{1}, \widetilde{x}_{2}} & \widetilde{c}_{11} \otimes \tilde{x}_{1} \oplus \widetilde{c}_{12} \otimes \widetilde{x}_{2} \\
\text { s.t. } & \left(\widetilde{x}_{1}, \widetilde{x}_{2}\right) \in \mathrm{IR} .
\end{array}
$$

Definition 8. A solution $\left(\widetilde{x}_{1}^{*}, \tilde{x}_{2}^{*}\right) \in \mathrm{IR}$ is said to be a fuzzy optimal solution of problem (11) if $\mathfrak{R}\left(\widetilde{c}_{11} \otimes \tilde{x}_{1}^{*} \oplus \widetilde{c}_{12} \otimes \tilde{x}_{2}^{*}\right) \geq$ $\Re\left(\widetilde{c}_{11} \otimes \widetilde{x}_{1} \oplus \widetilde{c}_{12} \otimes \widetilde{x}_{2}\right)$ for all solutions $\left(\widetilde{x}_{1}, \widetilde{x}_{2}\right) \in \operatorname{IR}$.

3.3. Transformed Problems Based on the Deviation Degree Measures and a Ranking Function Method. In this section, a new approach based on the deviation degree measures and a ranking function method of fuzzy numbers is developed to deal with problem (11). The main idea of our approach is to apply the deviation degree measures to deal with the fuzzy constraints, make use of a ranking function of fuzzy numbers to rank the upper and lower level fuzzy objective functions, and then transform problem (11) into a corresponding crisp bilevel programming problem.

For problem (11), we first convert fuzzy constraints into crisp ones by the deviation degree measures.

Clearly, the left-hand side of each constraint is a fuzzy number by using fuzzy arithmetic operations. Thus each constraint of problem (11) can be taken into account as the fuzzy comparisons between two fuzzy numbers. Here the positive deviation degree measure of two fuzzy numbers is applied to convert fuzzy constraints into crisp ones.

In terms of Definition 6, problem (11) can be formulated as

$$
\begin{array}{ll}
\max _{\widetilde{x}_{1}, \tilde{x}_{2}} & \tilde{c}_{11} \otimes \tilde{x}_{1} \oplus \tilde{c}_{12} \otimes \tilde{x}_{2} \\
& \text { where } \tilde{x}_{2} \text { solves } \\
\max _{\widetilde{x}_{2}} & \widetilde{c}_{21} \otimes \tilde{x}_{1} \oplus \tilde{c}_{22} \otimes \tilde{x}_{2} \\
\text { s.t. } & D_{\tilde{b}_{i}}\left(\left(\tilde{a}_{i 1} \otimes \tilde{x}_{1} \oplus \tilde{a}_{i 2} \otimes \tilde{x}_{2}\right)^{+}\right) \leq \delta_{i},
\end{array}
$$

$$
i=1,2, \ldots, m \text {, }
$$


where $\delta_{i}$ is an allowed deviation degree of $i$ th constraint, $i=$ $1,2, \ldots, m$, which is specified by the decision makers.

Let the elements of fuzzy vectors $\tilde{x}_{j}, \widetilde{c}_{l j}$, and $\tilde{a}_{i j}$ be represented by the triangular fuzzy numbers $\left(x_{j k}^{(1)}, x_{j k}^{(2)}\right.$, $\left.x_{j k}^{(3)}\right),\left(c_{l j k}^{(1)}, c_{l j k}^{(2)}, c_{l j k}^{(3)}\right)$ and $\left(a_{i j k}^{(1)}, a_{i j k}^{(2)}, a_{i j k}^{(3)}\right)$, respectively, $l=$ $1,2, j=1,2, i=1,2, \ldots, m$, and $k=1,2, \ldots, n_{j}$. Let triangular fuzzy numbers $\widetilde{b}_{i}=\left(b_{i}^{(1)}, b_{i}^{(2)}, b_{i}^{(3)}\right)$.

In terms of fuzzy arithmetic operations, we have

$$
\begin{aligned}
\tilde{a}_{i 1} \otimes \tilde{x}_{1} \oplus \tilde{a}_{i 2} \otimes \tilde{x}_{2}= & \sum_{k=1}^{n_{1}}\left(a_{i 1 k}^{(1)}, a_{i 1 k}^{(2)}, a_{i 1 k}^{(3)}\right) \\
& \otimes\left(x_{1 k}^{(1)}, x_{1 k}^{(2)}, x_{1 k}^{(3)}\right) \\
& \oplus \sum_{k=1}^{n_{2}}\left(a_{i 2 k}^{(1)}, a_{i 2 k}^{(2)}, a_{i 2 k}^{(3)}\right) \\
& \otimes\left(x_{2 k}^{(1)}, x_{2 k}^{(2)}, x_{2 k}^{(3)}\right) .
\end{aligned}
$$

Obviously, the left side of every constraint of problem (11) becomes a triangular fuzzy number. Let $\left(\widetilde{a}_{i 1} \otimes \widetilde{x}_{1} \oplus \tilde{a}_{i 2} \otimes \widetilde{x}_{2}\right)=$ $\left(a_{i}^{(1)}, a_{i}^{(2)}, a_{i}^{(3)}\right)$. From Definition 6, we have

$$
\begin{aligned}
D_{\tilde{b}_{i}} & \left(\left(\tilde{a}_{i 1} \otimes \tilde{x}_{1} \oplus \tilde{a}_{i 2} \otimes \tilde{x}_{2}\right)^{+}\right) \\
= & \frac{\sum_{h=1}^{3} \alpha^{(h)} \max \left(a_{i}^{(h)}-b_{i}^{(h)}, 0\right)}{\sum_{h=1}^{3} \alpha^{(h)} b_{i}^{(h)}} .
\end{aligned}
$$

Then problem (16) can be rewritten as

$$
\begin{gathered}
\max _{\left(x_{1 k_{1}}^{(1)}, x_{1 k_{1}}^{(2)}, x_{1 k_{1}}^{(3)}, x_{2 k_{2}}^{(1)}, x_{2 k_{2}}^{(2)}, x_{2 k_{2}}^{(3)}\right)} \sum_{k_{1}=1}^{n_{1}}\left(c_{11 k_{1}}^{(1)}, c_{11 k_{1}}^{(2)}, c_{11 k_{1}}^{(3)}\right) \\
\otimes\left(x_{1 k_{1}}^{(1)}, x_{1 k_{1}}^{(2)}, x_{1 k_{1}}^{(3)}\right) \\
\oplus \sum_{k_{2}=1}^{n_{2}}\left(c_{12 k_{2}}^{(1)}, c_{12 k_{2}}^{(2)}, c_{12 k_{2}}^{(3)}\right) \\
\otimes\left(x_{2 k_{2}}^{(1)}, x_{2 k_{2}}^{(2)}, x_{2 k_{2}}^{(3)}\right) \\
\text { where }\left(x_{2 k_{2}}^{(1)}, x_{2 k_{2}}^{(2)}, x_{2 k_{2}}^{(3)}\right) \text { solves } \\
\sum_{\left(x_{2 k_{2}}^{(1)}, x_{2 k_{2}}, x_{2 k_{2}}\right)}^{n_{1}}\left(c_{21 k_{1}}^{(1)}, c_{21 k_{1}}^{(2)}, c_{21 k_{1}}^{(3)}\right) \\
k_{1}=1 \\
\otimes\left(x_{1 k_{1}}^{(1)}, x_{1 k_{1}}^{(2)}, x_{1 k_{1}}^{(3)}\right) \\
\oplus \sum_{k_{2}=1}^{n_{2}}\left(c_{22 k_{2}}^{(1)}, c_{22 k_{2}}^{(2)}, c_{22 k_{2}}^{(3)}\right) \\
\otimes\left(x_{2 k_{2}}^{(1)}, x_{2 k_{2}}^{(2)}, x_{2 k_{2}}^{(3)}\right) \\
\sum_{h=1}^{3} \alpha^{(h)} \max _{i}\left(a_{i}^{(h)}-b_{i}^{(h)}, 0\right) \\
\leq \delta_{i} \sum_{h=1}^{3} \alpha^{(h)} b_{i}^{(h)}, \\
\text { s.t. }
\end{gathered}
$$

where $x_{1 k_{1}}^{(1)}, x_{1 k_{1}}^{(2)}, x_{1 k_{1}}^{(3)}, x_{2 k_{2}}^{(1)}, x_{2 k_{2}}^{(2)}$, and $x_{2 k_{2}}^{(3)}$ are nonnegative.
It is clear that the constraints of problem (19) are nonlinear. To make calculation easy, the variable substitution is applied to convert nonlinear constraints into linear ones.

Let $u_{i}^{(h)}=\max \left(a_{i}^{(h)}-b_{i}^{(h)}, 0\right), h=1,2,3$ and $i=$ $1,2, \ldots, m$. Then problem (19) can be equivalently transformed into the following problem:

$$
\begin{aligned}
\max _{\left(x_{1 k_{1}}^{(1)}, x_{1 k_{1}}^{(2)}, x_{1 k_{1}}^{(3)}, x_{2 k_{2}}^{(1)}, x_{2 k_{2}}^{(2)}, x_{2 k_{2}}^{(3)}\right)} & \sum_{k_{1}=1}^{n_{1}}\left(c_{11 k_{1}}^{(1)}, c_{11 k_{1}}^{(2)}, c_{11 k_{1}}^{(3)}\right) \\
& \otimes\left(x_{1 k_{1}}^{(1)}, x_{1 k_{1}}^{(2)}, x_{1 k_{1}}^{(3)}\right) \\
& \oplus \sum_{k_{2}=1}^{n_{2}}\left(c_{12 k_{2}}^{(1)}, c_{12 k_{2}}^{(2)}, c_{12 k_{2}}^{(3)}\right) \\
& \otimes\left(x_{2 k_{2}}^{(1)}, x_{2 k_{2}}^{(2)}, x_{2 k_{2}}^{(3)}\right)
\end{aligned}
$$

where $\left(x_{2 k_{2}}^{(1)}, x_{2 k_{2}}^{(2)}, x_{2 k_{2}}^{(3)}\right)$ solves

$$
\begin{aligned}
\max _{\left(x_{2 k_{2}}^{(1)}, x_{2 k_{2}}^{(2)}, x_{2 k_{2}}^{(3)}\right)} & \sum_{k_{1}=1}^{n_{1}}\left(c_{21 k_{1}}^{(1)}, c_{21 k_{1}}^{(2)}, c_{21 k_{1}}^{(3)}\right) \\
& \otimes\left(x_{1 k_{1}}^{(1)}, x_{1 k_{1}}^{(2)}, x_{1 k_{1}}^{(3)}\right) \\
& \oplus \sum_{k_{2}=1}^{n_{2}}\left(c_{22 k_{2}}^{(1)}, c_{22 k_{2}}^{(2)}, c_{22 k_{2}}^{(3)}\right) \\
& \otimes\left(x_{2 k_{2}}^{(1)}, x_{2 k_{2}}^{(2)}, x_{2 k_{2}}^{(3)}\right)
\end{aligned}
$$$$
\text { s.t. } \quad \sum_{h=1}^{3} \alpha^{(h)} u_{i}^{(h)} \leq \delta_{i} \sum_{h=1}^{3} \alpha^{(h)} b_{i}^{(h)} \text {, }
$$$$
i=1,2, \ldots, m,
$$$$
a_{i}^{(h)}-b_{i}^{(h)} \leq u_{i}^{(h)},
$$$$
h=1,2,3, i=1,2, \ldots, m
$$$$
u_{i}^{(h)} \geq 0 \text {, }
$$$$
h=1,2,3, i=1,2, \ldots, m,
$$

$x_{1 k_{1}}^{(1)} \geq 0$,

$x_{1 k_{1}}^{(2)}-x_{1 k_{1}}^{(1)} \geq 0$,

$x_{1 k_{1}}^{(3)}-x_{1 k_{1}}^{(2)} \geq 0$,

$x_{2 k_{2}}^{(1)} \geq 0$,

$x_{2 k_{2}}^{(2)}-x_{2 k_{2}}^{(1)} \geq 0$,

$x_{2 k_{2}}^{(3)}-x_{2 k_{2}}^{(2)} \geq 0$.

It is worth mentioning that problem (20) is a bilevel linear programming problem with triangular fuzzy coefficients and triangular fuzzy decision variables in both objective functions. It is well known that a ranking function is a popular approach for solving a fully fuzzy programming problem. Next, we use a ranking function method to convert the upper and lower level objective functions into 
the corresponding crisp ones. Hence, problem (20) can be transformed as

$$
\begin{aligned}
& \max _{\left(x_{1 k_{1}}^{(1)}, x_{1 k_{1}}^{(2)}, x_{1 k_{1}}^{(3)}, x_{2 k_{2}}^{(1)}, x_{2 k_{2}}^{(2)}, x_{2 k_{2}}^{(3)}\right)} \mathfrak{R}\left(\sum_{k_{1}=1}^{n_{1}}\left(c_{11 k_{1}}^{(1)}, c_{11 k_{1}}^{(2)}, c_{11 k_{1}}^{(3)}\right) \otimes\left(x_{1 k_{1}}^{(1)}, x_{1 k_{1}}^{(2)}, x_{1 k_{1}}^{(3)}\right) \oplus \sum_{k_{2}=1}^{n_{2}}\left(c_{12 k_{2}}^{(1)}, c_{12 k_{2}}^{(2)}, c_{12 k_{2}}^{(3)}\right) \otimes\left(x_{2 k_{2}}^{(1)}, x_{2 k_{2}}^{(2)}, x_{2 k_{2}}^{(3)}\right)\right) \\
& \text { where }\left(x_{2 k_{2}}^{(1)}, x_{2 k_{2}}^{(2)}, x_{2 k_{2}}^{(3)}\right) \text { solves } \\
& \max _{\left(x_{2 k_{2}}^{(1)}, x_{2 k_{2}}^{(2)}, x_{2 k_{2}}^{(3)}\right)} \mathfrak{R}\left(\sum_{k_{1}=1}^{n_{1}}\left(c_{21 k_{1}}^{(1)}, c_{21 k_{1}}^{(2)}, c_{21 k_{1}}^{(3)}\right) \otimes\left(x_{1 k_{1}}^{(1)}, x_{1 k_{1}}^{(2)}, x_{1 k_{1}}^{(3)}\right) \oplus \sum_{k_{2}=1}^{n_{2}}\left(c_{22 k_{2}}^{(1)}, c_{22 k_{2}}^{(2)}, c_{22 k_{2}}^{(3)}\right) \otimes\left(x_{2 k_{2}}^{(1)}, x_{2 k_{2}}^{(2)}, x_{2 k_{2}}^{(3)}\right)\right) \\
& \text { s.t. } \quad \sum_{h=1}^{3} \alpha^{(h)} u_{i}^{(h)} \leq \delta_{i} \sum_{h=1}^{3} \alpha^{(h)} b_{i}^{(h)}, \quad i=1,2, \ldots, m \\
& a_{i}^{(h)}-b_{i}^{(h)} \leq u_{i}^{(h)}, \quad h=1,2,3, i=1,2, \ldots, m \\
& u_{i}^{(h)} \geq 0, \quad h=1,2,3, \quad i=1,2, \ldots, m \\
& x_{1 k_{1}}^{(1)} \geq 0 \text {, } \\
& x_{1 k_{1}}^{(2)}-x_{1 k_{1}}^{(1)} \geq 0 \text {, } \\
& x_{1 k_{1}}^{(3)}-x_{1 k_{1}}^{(2)} \geq 0 \text {, } \\
& x_{2 k_{2}}^{(1)} \geq 0 \text {, } \\
& x_{2 k_{2}}^{(2)}-x_{2 k_{2}}^{(1)} \geq 0 \text {, } \\
& x_{2 k_{2}}^{(3)}-x_{2 k_{2}}^{(2)} \geq 0 \text {. }
\end{aligned}
$$

Obviously, problem (21) is a crisp bilevel linear programming problem.

Proposition 9. For a given allowed deviation degree $\delta=$ $\left(\delta_{1}, \delta_{2}, \ldots, \delta_{m}\right)$, if $x_{1 k_{1}}^{(1)}, x_{1 k_{1}}^{(2)}, x_{1 k_{1}}^{(3)}, k_{1}=1,2, \ldots, n_{1}, x_{2 k_{2}}^{(1)}$, $x_{2 k_{2}}^{(2)}, x_{2 k_{2}}^{(3)}, k_{2}=1,2, \ldots, n_{2}$, are an optimal solution of problem (21) and letting $\tilde{x}_{1 k_{1}}=\left(x_{1 k_{1}}^{(1)}, x_{1 k_{1}}^{(2)}, x_{1 k_{1}}^{(3)}\right), \tilde{x}_{2 k_{2}}=$ $\left(x_{2 k_{2}}^{(1)}, x_{2 k_{2}}^{(2)}, x_{2 k_{2}}^{(3)}\right)$, then $\tilde{x}_{1}=\left(\tilde{x}_{11}, \tilde{x}_{12}, \ldots, \tilde{x}_{1 k_{1}}, \ldots, \tilde{x}_{1 n_{1}}\right), \tilde{x}_{2}=$ $\left(\widetilde{x}_{21}, \tilde{x}_{22}, \ldots, \tilde{x}_{2 k_{2}}, \ldots, \tilde{x}_{1 n_{2}}\right)$ are a fuzzy optimal solution of problem (11).

Proposition 9 means that the most direct approach for solving problem (11) is to solve the equivalent mathematical programming problem (21). One advantage that it offers is that it allows for a more robust model to be solved without introducing any new computational difficulties.

Let $\bar{S}$ denote the constraint region of problem (21). Then problem (21) can be transformed into the following problem:

$$
\begin{aligned}
& \max _{\left(x_{1 k_{1}}^{(1)}, x_{1 k_{1}}^{(2)}, x_{1 k_{1}}^{(3)}, x_{2 k_{2}}^{(1)}, x_{2 k_{2}}^{(2)}, x_{2 k_{2}}^{(3)}\right)} \Re\left(\sum_{k_{1}=1}^{n_{1}}\left(c_{11 k_{1}}^{(1)}, c_{11 k_{1}}^{(2)}, c_{11 k_{1}}^{(3)}\right) \otimes\left(x_{1 k_{1}}^{(1)}, x_{1 k_{1}}^{(2)}, x_{1 k_{1}}^{(3)}\right) \oplus \sum_{k_{2}=1}^{n_{2}}\left(c_{12 k_{2}}^{(1)}, c_{12 k_{2}}^{(2)}, c_{12 k_{2}}^{(3)}\right) \otimes\left(x_{2 k_{2}}^{(1)}, x_{2 k_{2}}^{(2)}, x_{2 k_{2}}^{(3)}\right)\right) \\
& \text { where }\left(x_{2 k_{2}}^{(1)}, x_{2 k_{2}}^{(2)}, x_{2 k_{2}}^{(3)}\right) \text { solves } \\
& \max _{\left(x_{2 k_{2}}^{(1)}, x_{2 k_{2}}^{(2)}, x_{2 k_{2}}^{(3)}\right)} \Re\left(\sum_{k_{1}=1}^{n_{1}}\left(c_{21 k_{1}}^{(1)}, c_{21 k_{1}}^{(2)}, c_{21 k_{1}}^{(3)}\right) \otimes\left(x_{1 k_{1}}^{(1)}, x_{1 k_{1}}^{(2)}, x_{1 k_{1}}^{(3)}\right) \oplus \sum_{k_{2}=1}^{n_{2}}\left(c_{22 k_{2}}^{(1)}, c_{22 k_{2}}^{(2)}, c_{22 k_{2}}^{(3)}\right) \otimes\left(x_{2 k_{2}}^{(1)}, x_{2 k_{2}}^{(2)}, x_{2 k_{2}}^{(3)}\right)\right) \\
& \text { s.t. } \quad\left(x_{1 k_{1}}^{(1)}, x_{1 k_{1}}^{(2)}, x_{1 k_{1}}^{(3)}, x_{2 k_{2}}^{(1)}, x_{2 k_{2}}^{(2)}, x_{2 k_{2}}^{(3)}\right) \in \bar{S} .
\end{aligned}
$$


Thus we can obtain a fuzzy optimal solution of problem (11) by solving problem (22).

3.4. The Computational Procedures for Finding the Fuzzy Optimal Solution of Problem (11). For problem (22), its feasible region relies on the allowed deviation degree $\delta$. It is obvious that different values of the allowed deviation degree produce different feasible regions and different upper level objective function values. In detail, when value of the allowed deviation degree is larger, the feasible region is larger and the upper level objective function value becomes higher. On the contrary, when value of the allowed deviation degree is smaller, the feasible region is also smaller and the upper level objective function value becomes lower. Thus there is a conflict of improving the upper level objective function value and decreasing the allowed deviation degree for the decision makers. To address this issue, we give a computational procedure for obtaining a fuzzy optimal solution of problem (11) by taking into account the overall balance between the above conflicts.

Step 1. Determine the allowed minimum and maximum deviation degrees of each constraint of problem (22).

For $i$ th constraint $(i=1,2,3, \ldots, m)$, its allowed maximum deviation $\delta_{i}^{R}$ is specified by the decision makers. In order to calculate the allowed minimum deviation $\delta_{i}^{L}$, solve the following problem:

$$
\begin{array}{ll}
\min & \sum_{i=1}^{m} \delta_{i} \\
\text { s.t. } & \left(x_{1 k_{1}}^{(1)}, x_{1 k_{1}}^{(2)}, x_{1 k_{1}}^{(3)}, x_{2 k_{2}}^{(1)}, x_{2 k_{2}}^{(2)}, x_{2 k_{2}}^{(3)}\right) \in \bar{S} .
\end{array}
$$

The allowed minimum and maximum deviation degrees of all constraints can be denoted by $\delta^{L}=\left(\delta_{1}^{L}, \delta_{2}^{L}, \ldots, \delta_{m}^{L}\right)$ and $\delta^{R}=\left(\delta_{1}^{R}, \delta_{2}^{R}, \ldots, \delta_{m}^{R}\right)$, respectively.

Step 2. Calculate $\delta^{L}$-fuzzy optimal solution and $\delta^{R}$-fuzzy optimal solution of problem (11).

For the values of $\delta^{L}$, solve problem (22). Denote its optimal solution by $\tilde{x}_{1 k_{1}}\left(\delta^{L}\right)=\left(x_{1 k_{1}}^{(1)}\left(\delta^{L}\right), x_{1 k_{1}}^{(2)}\left(\delta^{L}\right), x_{1 k_{1}}^{(3)}\left(\delta^{L}\right)\right)$, $k_{1}=1,2, \ldots, n_{1}, \tilde{x}_{2 k_{2}}\left(\delta^{L}\right)=\left(x_{2 k_{2}}^{(1)}\left(\delta^{L}\right), x_{2 k_{2}}^{(2)}\left(\delta^{L}\right), x_{2 k_{2}}^{(3)}\left(\delta^{L}\right)\right)$, $k_{2}=1,2, \ldots, n_{2}$. Then the optimal solution $\tilde{x}_{1}\left(\delta^{L}\right)=$ $\left(\widetilde{x}_{11}\left(\delta^{L}\right), \tilde{x}_{12}\left(\delta^{L}\right), \ldots, \tilde{x}_{1 k_{1}}\left(\delta^{L}\right), \ldots, \tilde{x}_{1 n_{1}}\left(\delta^{L}\right)\right), \tilde{x}_{2}\left(\delta^{L}\right)=$ $\left(\tilde{x}_{21}\left(\delta^{L}\right), \tilde{x}_{22}\left(\delta^{L}\right), \ldots, \tilde{x}_{2 k_{2}}\left(\delta^{L}\right), \ldots, \tilde{x}_{1 n_{2}}\left(\delta^{L}\right)\right)$ is a $\delta^{L}$-fuzzy optimal solution of problem (11). Using fuzzy arithmetic, we can obtain the corresponding upper and lower fuzzy objective values denoted by $\widetilde{Z}_{1}^{*}\left(\delta^{L}\right)=\left(\widetilde{z}_{1}^{*(1)}\left(\delta^{L}\right), \widetilde{z}_{1}^{*(2)}\left(\delta^{L}\right), \widetilde{z}_{1}^{*(3)}\left(\delta^{L}\right)\right)$ and $\widetilde{Z}_{2}^{*}\left(\delta^{L}\right)=\left(\widetilde{z}_{2}^{*(1)}\left(\delta^{L}\right), \widetilde{z}_{2}^{*(2)}\left(\delta^{L}\right), \widetilde{z}_{2}^{*(3)}\left(\delta^{L}\right)\right)$.

Similarly, for the values of $\delta^{R}$, we solve problem (22). Let $\tilde{x}_{1 k_{1}}\left(\delta^{R}\right)=\left(x_{1 k_{1}}^{(1)}\left(\delta^{R}\right), x_{1 k_{1}}^{(2)}\left(\delta^{R}\right), x_{1 k_{1}}^{(3)}\left(\delta^{R}\right)\right), k_{1}=1,2, \ldots, n_{1}$, $\tilde{x}_{2 k_{2}}\left(\delta^{R}\right)=\left(x_{2 k_{2}}^{(1)}\left(\delta^{R}\right), x_{2 k_{2}}^{(2)}\left(\delta^{R}\right), x_{2 k_{2}}^{(3)}\left(\delta^{R}\right)\right), k_{2}=1,2, \ldots, n_{2}$, denote its optimal solution to problem (22). Then the optimal solution $\tilde{x}_{1}\left(\delta^{R}\right)=\left(\widetilde{x}_{11}\left(\delta^{R}\right), \widetilde{x}_{12}\left(\delta^{R}\right), \ldots, \widetilde{x}_{1 k_{1}}\left(\delta^{R}\right)\right.$, $\left.\ldots, \tilde{x}_{1 n_{1}}\left(\delta^{R}\right)\right)$ and $\tilde{x}_{2}\left(\delta^{R}\right)=\left(\tilde{x}_{21}\left(\delta^{R}\right), \tilde{x}_{22}\left(\delta^{R}\right), \ldots, \tilde{x}_{2 k_{2}}\left(\delta^{R}\right)\right.$, $\left.\ldots, \tilde{x}_{1 n_{2}}\left(\delta^{R}\right)\right)$ is a $\delta^{R}$-fuzzy optimal solution of problem (11).
Correspondingly the upper and lower fuzzy objective values denoted by $\widetilde{Z}_{1}^{*}\left(\delta^{R}\right)=\left(\widetilde{z}_{1}^{*(1)}\left(\delta^{R}\right), \widetilde{z}_{1}^{*(2)}\left(\delta^{R}\right), \widetilde{z}_{1}^{*(3)}\left(\delta^{R}\right)\right)$, $\widetilde{Z}_{2}^{*}\left(\delta^{R}\right)=\left(\widetilde{z}_{2}^{*(1)}\left(\delta^{R}\right), \widetilde{z}_{2}^{*(2)}\left(\delta^{R}\right), \widetilde{z}_{2}^{*(3)}\left(\delta^{R}\right)\right)$ of problem (11) can be obtained.

Step 3. Determine the membership functions of the upper and lower level objective functions.

In order to obtain a fuzzy optimal solution which balances a conflict of improving the upper level objective function value and decreasing the allowed deviation degree, we elicit the membership functions of fuzzy goals for each of the objective functions. Although the membership function does not always need to be linear, for the sake of simplicity, the linear membership functions for the upper and lower level objective functions may be given as follows:

$$
\mu\left(z_{k}^{(h)}\right)= \begin{cases}0, & z_{k}^{(h)} \leq z_{k \min }^{(h)}, \\ \frac{z_{k}^{(h)}-z_{k \min }^{(h)}}{z_{k \max }^{(h)}-z_{k \min }^{(h)},} & z_{k \min }^{(h)} \leq z_{k}^{(h)} \leq z_{k \max }^{(h)} \\ 1, & z^{(h)} \geq z_{k \max }^{(h)}\end{cases}
$$

where $z_{k \max }^{(h)}=\max \left\{\widetilde{z}_{k}^{*(h)}\left(\delta^{L}\right), \widetilde{z}_{k}^{*(h)}\left(\delta^{R}\right)\right\}$ and $z_{k \min }^{(h)}=$ $\min \left\{\widetilde{z}_{k}^{*(h)}\left(\delta^{L}\right), \widetilde{z}_{k}^{*(h)}\left(\delta^{R}\right)\right\}, k=1,2$ and $h=1,2,3$.

Step 4. Calculate the fuzzy optimal solution of problem (11).

Taking into account the balance between a better upper level objective function value and a smaller deviation degree, we introduce max-min operation to construct a model. To do so, we introduce an auxiliary variable $\lambda$ and construct the following optimization problem:

$$
\begin{array}{ll}
\max & \lambda \\
\text { s.t. } & \left(z_{1 \max }^{(h)}-z_{1 \min }^{(h)}\right) \lambda \leq z_{1}^{(h)}-z_{1 \mathrm{~min}}^{(h)}, \quad h=1,2,3, \\
& \left(z_{2 \max }^{(h)}-z_{2 \min }^{(h)}\right) \lambda \leq z_{2}^{(h)}-z_{2 \min }^{(h)}, \quad h=1,2,3, \\
& \delta_{i}=\lambda \delta_{i}^{L}+(1-\lambda) \delta_{i}^{R}, \quad i=1,2, \ldots, m, \\
& 0 \leq \lambda \leq 1, \\
& \left(x_{1 k_{1}}^{(1)}, x_{1 k_{1}}^{(2)}, x_{1 k_{1}}^{(3)}, x_{2 k_{2}}^{(1)}, x_{2 k_{2}}^{(2)}, x_{2 k_{2}}^{(3)}\right) \in S .
\end{array}
$$

By solving the above problem, we can find the fuzzy optimal solution of problem (11).

As a consequence, a fuzzy optimal solution of problem (11) can be obtained in terms of the above computation method.

\section{Experiment Results and Analysis}

In this section, a numerical example is considered to demonstrate the feasibility of the proposed approach for solving a fully fuzzy bilevel linear programming problem. In addition, we make some comparisons on the performance of the proposed approach and the existing method developed by Safaei and Saraj [28]. 
4.1. Numerical Example. Consider the following fully fuzzy bilevel linear programming problem in [28]:

$$
\begin{array}{ll}
\max _{\widetilde{x}_{1}} & \widetilde{Z}_{1}=(3,5,7) \otimes \tilde{x}_{1} \oplus(2,4,8) \otimes \tilde{x}_{2} \\
& \text { where } \tilde{x}_{2} \text { solves } \\
\max _{\widetilde{x}_{2}} & \widetilde{Z}_{2}=(3,5,10) \otimes \tilde{x}_{1} \oplus(1,7,8) \otimes \tilde{x}_{2} \\
\text { s.t. } & (4,5,9) \otimes \tilde{x}_{1} \oplus(2,7,8) \otimes \tilde{x}_{2} \leq(4,10,20), \\
& (0,3,7) \otimes \tilde{x}_{1} \oplus(1,2,10) \otimes \tilde{x}_{2} \leq(2,5,18), \\
& \tilde{x}_{1}, \tilde{x}_{2} \geq \tilde{0},
\end{array}
$$

where $\tilde{x}_{1}, \tilde{x}_{2}$ are triangular fuzzy numbers.

Assume that $\tilde{x}_{1}=\left(x_{1}^{(1)}, x_{1}^{(2)}, x_{1}^{(3)}\right)$ and $\tilde{x}_{2}=\left(x_{2}^{(1)}, x_{2}^{(2)}\right.$, $\left.x_{2}^{(3)}\right)$. According to arithmetic operations of triangular fuzzy numbers, the fully fuzzy bilevel programming problem (26) can be rewritten as

$$
\begin{aligned}
\max _{\left(x_{1}^{(1)}, x_{1}^{(2)}, x_{1}^{(3)}\right)} & \left(3 x_{1}^{(1)}+2 x_{2}^{(1)}, 5 x_{1}^{(2)}+4 x_{2}^{(2)}, 7 x_{1}^{(3)}+8 x_{2}^{(3)}\right) \\
& \text { where }\left(x_{2}^{(1)}, x_{2}^{(2)}, x_{2}^{(3)}\right) \text { solve } \\
\max _{\left(x_{2}^{(1)}, x_{2}^{(2)}, x_{2}^{(3)}\right)} & \left(3 x_{1}^{(1)}+x_{2}^{(1)}, 5 x_{1}^{(2)}+7 x_{2}^{(2)}, 10 x_{1}^{(3)}+8 x_{2}^{(3)}\right) \\
\text { s.t. } \quad & \left(4 x_{1}^{(1)}+2 x_{2}^{(1)}, 5 x_{1}^{(2)}+7 x_{2}^{(2)}, 9 x_{1}^{(3)}+8 x_{2}^{(3)}\right) \\
& \leq(4,10,20), \\
& \left(x_{2}^{(1)}, 3 x_{1}^{(2)}+2 x_{2}^{(2)}, 7 x_{1}^{(3)}+10 x_{2}^{(3)}\right) \\
& (2,5,18), \\
& x_{1}^{(1)} \geq 0, \\
& x_{1}^{(2)}-x_{1}^{(1)} \geq 0, \\
& x_{1}^{(3)}-x_{1}^{(2)} \geq 0 \\
& x_{2}^{(1)} \geq 0, \\
& x_{2}^{(2)}-x_{2}^{(1)} \geq 0 \\
& x_{2}^{(3)}-x_{2}^{(2)} \geq 0 \\
&
\end{aligned}
$$

where $\left(x_{1}^{(1)}, x_{1}^{(2)}, x_{1}^{(3)}\right),\left(x_{2}^{(1)}, x_{2}^{(2)}, x_{2}^{(3)}\right)$ are triangular fuzzy numbers.

4.2. Solution Processes and Results. Based on the proposed models and approach, the procedure for finding the fuzzy optimal solution of the fully fuzzy bilevel linear programming problem (26) is described as follows.

Step 1. According to model (23), the allowed minimum deviation degree can be calculated and the obtained optimal solution is $\delta^{L}=\left(\delta_{1}^{L}, \delta_{2}^{L}\right)=(0,0)$. Here the allowed maximum deviation deviation is specified by the decision makers, and suppose that the decision makers set the allowed maximum deviation deviation $\delta^{R}=\left(\delta_{1}^{R}, \delta_{2}^{R}\right)=(0.05,0.05)$.

Step 2. By model (22), for $\delta^{L}=\left(\delta_{1}^{L}, \delta_{2}^{L}\right)=(0,0)$, we can obtain the optimal solution $x_{1}^{(1)}=0.7727, x_{1}^{(2)}=$ $1.3636, x_{1}^{(3)}=1.5294, x_{2}^{(1)}=0.4545, x_{2}^{(2)}=0.4545, x_{2}^{(3)}=$ 0.6471 . Thus the $\delta^{L}$-fuzzy optimal solution to problem $(26)$ is $\tilde{x}_{1}^{*}\left(\delta^{L}\right)=(0.7727,1.3636,1.5294), \tilde{x}_{2}^{*}\left(\delta^{L}\right)=$ $(0.4545,0.4545,0.6471)$, and the corresponding upper and lower level fuzzy objective function values are $\widetilde{Z}_{1}^{*}\left(\delta^{L}\right)=$ $(3.2237,8.6314,16.7059), \widetilde{Z}_{2}^{*}\left(\delta^{L}\right)=(2.7727,10,21.6471)$.

Similarly, for $\delta^{R}=\left(\delta_{1}^{R}, \delta_{2}^{R}\right)=(0.05,0.05)$, we can obtain the optimal solution as $x_{1}^{(1)}=1.3284, x_{1}^{(2)}=$ $1.8409, x_{1}^{(3)}=1.8409, x_{2}^{(1)}=0.1136, x_{2}^{(2)}=0.1136, x_{2}^{(3)}=$ 0.5114 . Thus the $\delta^{R}$-fuzzy optimal solution to problem $(26)$ is $\tilde{x}_{1}^{*}\left(\delta^{L}\right)=(1.3284,1.8409,1.8409), \tilde{x}_{2}^{*}\left(\delta^{L}\right)=$ $(0.1136,0.1136,0.5114)$, and the corresponding upper and lower level fuzzy objective function values are $\widetilde{Z}_{1}^{*}\left(\delta^{L}\right)=$ $(4.2125,9.6591,16.9773), \widetilde{Z}_{2}^{*}\left(\delta^{L}\right)=(4.0989,10,22.5)$.

Step 3. The linear membership functions of the upper and lower level objective functions can be described as follows:

$$
\begin{aligned}
& \mu\left(z_{1}^{(1)}\right) \\
& = \begin{cases}0, & z_{1}^{(1)} \leq 3.2237, \\
\frac{z_{1}^{(1)}-3.2273}{4.2125-3.2273}, & 3.2237 \leq z_{1}^{(1)} \leq 4.2125, \\
1, & z_{1}^{(1)} \geq 4.2125,\end{cases} \\
& \mu\left(z_{1}^{(2)}\right) \\
& = \begin{cases}0, & z_{1}^{(2)} \leq 8.6314, \\
\frac{z_{1}^{(2)}-8.6314}{9.6591-8.6314}, & 8.6314 \leq z_{1}^{(2)} \leq 9.6591, \\
1, & z_{1}^{(2)} \geq 9.6591,\end{cases} \\
& \mu\left(z_{1}^{(3)}\right) \\
& = \begin{cases}0, & z_{1}^{(3)} \leq 16.7059, \\
\frac{z_{1}^{(3)}-16.7059}{16.9773-16.7059}, & 16.7059 \leq z_{1}^{(3)} \leq 16.9773, \\
1, & z_{1}^{(3)} \geq 16.97731,\end{cases} \\
& \mu\left(z_{2}^{(1)}\right) \\
& = \begin{cases}0, & z_{2}^{(1)} \leq 2.7727, \\
\frac{z_{2}^{(1)}-2.7727}{4.0989-2.7727}, & 2.7727 \leq z_{2}^{(1)} \leq 4.0989, \\
1, & z_{2}^{(1)} \geq 4.0989,\end{cases}
\end{aligned}
$$




$$
\begin{aligned}
& \mu\left(z_{2}^{(3)}\right) \\
& = \begin{cases}0, & z_{2}^{(3)} \leq 21.6471, \\
\frac{z_{2}^{(3)}-21.6471}{22.5-21.6471}, & 21.6471 \leq z_{2}^{(3)} \leq 22.5, \\
1, & z_{2}^{(3)} \geq 22.5 .\end{cases}
\end{aligned}
$$

Step 4. According to model (25), the fuzzy optimal solution of problem (26) can be obtained by solving the following problem:

$$
\begin{array}{ll}
\max & \lambda \\
\text { s.t. } & 0.9852 \lambda \leq z_{1}^{(1)}-3.2273, \\
& 1.0277 \lambda \leq z_{1}^{(2)}-8.6314, \\
& 0.2714 \lambda \leq z_{1}^{(3)}-16.7059, \\
& 1.3262 \lambda \leq z_{2}^{(1)}-2.7727, \\
& z_{2}^{(2)}=10, \\
& 0.8529 \lambda \leq z_{2}^{(3)}-21.6471, \\
& \delta_{1}=0.05(1-\lambda), \\
& \delta_{2}=0.05(1-\lambda), \\
& 0 \leq \lambda \leq 1, \\
& x_{1}^{(1)}, x_{1}^{(2)}, x_{1}^{(3)}, x_{2}^{(1)}, x_{2}^{(2)}, x_{2}^{(3)} \in S .
\end{array}
$$

The optimal solution of problem (29) is $\delta_{1}^{*}=0.025, \delta_{2}^{*}=$ $0.025, \lambda^{*}=0.5, x_{1}^{(1)}=1.0506, x_{1}^{(2)}=1.6014, x_{1}^{(3)}=$ $1.7440, x_{2}^{(1)}=0.2841, x_{2}^{(2)}=0.2847, x_{2}^{(3)}=0.5792, z_{1}^{(1)}=$ $3.7199, z_{1}^{(2)}=9.1458, z_{1}^{(3)}=16.8416, z_{2}^{(1)}=3.4358, z_{2}^{(2)}=$ $10, z_{2}^{(3)}=22.0735$. That is, the fuzzy optimal solution of problem $(26)$ is $\widetilde{x}_{1}^{*}\left(\delta^{*}\right)=(1.0506,1.6014,1.7440), \tilde{x}_{2}^{*}\left(\delta^{*}\right)=$ $(0.2841,0.2847,0.5792)$. Correspondingly, the upper and lower level fuzzy objective function values are $\widetilde{Z}_{1}^{*}\left(\delta^{*}\right)=$ $(3.7199,9.1458,16.8416), \widetilde{Z}_{2}^{*}\left(\delta^{*}\right)=(3.4358,10,22.0735)$, respectively.

4.3. Comparisons with the Existing Method. To show the advantages of our proposed method, in this section, we make some comparisons of the results obtained by the existing approach and by our proposed approach.

For a fully fuzzy bilevel programming problem, Safaei and Saraj [28] developed a bound and decomposition method to deal with such a problem. To find the optimal solution, this approach required decomposing the fully fuzzy bilevel programming problem into three crisp linear programming problems with bounded variables (middle level bilevel problem, upper level bilevel problem, and lower level bilevel problem). Nevertheless, this approach may not be practical

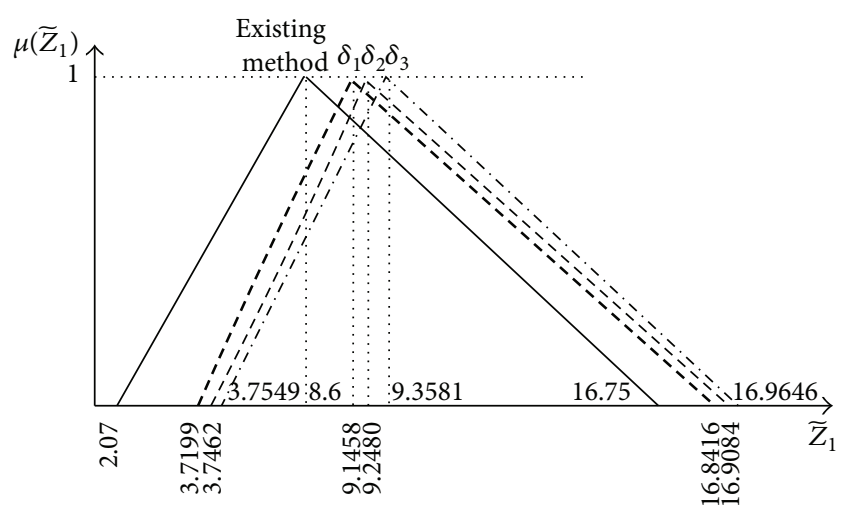

FIGURE 1: The membership function of the upper level fuzzy objective value provided by our approach and the existing method.

for complex problems in the real world. Unlike Safaei and Saraj's approach, the final transformed model by the proposed approach is only a bilevel linear programming model which is the simplest case in bilevel programming. Obviously, our approach requires relatively low computation.

Table 1 provides the results obtained by the proposed approach and the existing approach [28]. Using Safaei and Saraj's approach [28] to deal with this example, the resulting fuzzy optimal solution is $\tilde{x}_{1}^{*}=(0.39,1.36,1.65)$, $\tilde{x}_{2}^{*}=(0.45,0.45,0.45)$, and the upper and lower level fuzzy objective function values are $\widetilde{Z}_{1}^{*}=(2.07,8.6,16.75)$, $\widetilde{Z}_{2}^{*}=(1.62,9.95,21.7)$. Since the problem is a maximization problem, it is desirable for the upper and lower level decision makers to obtain a big objective function value. Obviously, our approach provides better upper and lower level objective function values compared with Safaei and Saraj's method. Thus it is clear that our method gives a better solution than the existing approaches in terms of the objective function values.

Table 2 presents the results of this example for different allowed maximum deviation degrees. Figure 1 gives the membership function of the upper level fuzzy objective function which is obtained by our approach under $\delta=$ $(0.05,0.05),(0.06,0.06)$, and $(0.07,0.07)$ and the existing method. It is clearly seen from Figure 1 that our method for different allowed maximum deviation degrees provides better fuzzy objective value than Safaei and Saraj's method. Thus our method outperforms the existing method. The membership function of the lower level fuzzy objective which is obtained by our approach and the existing method is given in Figure 2 . As it can be seen from Figure 2 our approach outforms the existing method in terms of the lower level fuzzy objective function values.

4.4. Sensitivity Analysis. In this section, we discuss variation in the objective function values with the change of the allowed maximum deviation degrees $\delta^{R}$. It can be seen from Table 2 that different $\delta^{R}$ would correspond to different lower bounds, most possible values, and upper bounds of the objective functions. With the increase of $\delta^{R}$, there is an increase of the lower bounds of the upper and lower level objective functions 
TABLE 1: Results obtained by our approach and the existing approach.

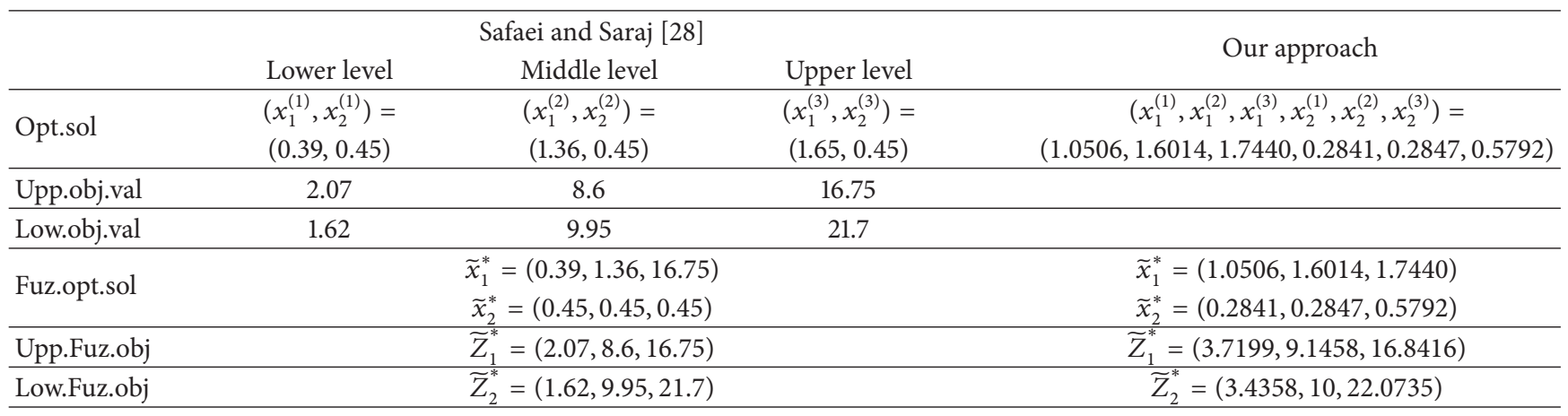

TABLE 2: The optimal solutions with different allowed maximum deviation degrees.

\begin{tabular}{lccr}
\hline & $\delta=(0.05,0.05)$ & $\delta=(0.06,0.06)$ & $\delta=(0.07,0.07)$ \\
\hline$\widetilde{x}_{1}^{*}\left(\delta^{*}\right)$ & $(1.0506,1.6014,1.7440)$ & $(1.0820,1.6491,1.7917)$ & $(1.0934,1.6894,1.8319)$ \\
$\widetilde{x}_{2}^{*}\left(\delta^{*}\right)$ & $(0.2841,0.2847,0.5792)$ & $(0.2500,0.2507,0.5458)$ & $(0.2273,0.2278,0.5177)$ \\
$\widetilde{Z}_{1}^{*}\left(\delta^{*}\right)$ & $(3.7199,9.1458,16.8416)$ & $(3.7462,9.2480,16.9084)$ & $(3.7549,9.3581,16.9646)$ \\
$\widetilde{Z}_{2}^{*}\left(\delta^{*}\right)$ & $(3.4358,10,22.0735)$ & $(3.4961,10,22.2835)$ & $(3.5076,10,22.4602)$ \\
\hline
\end{tabular}

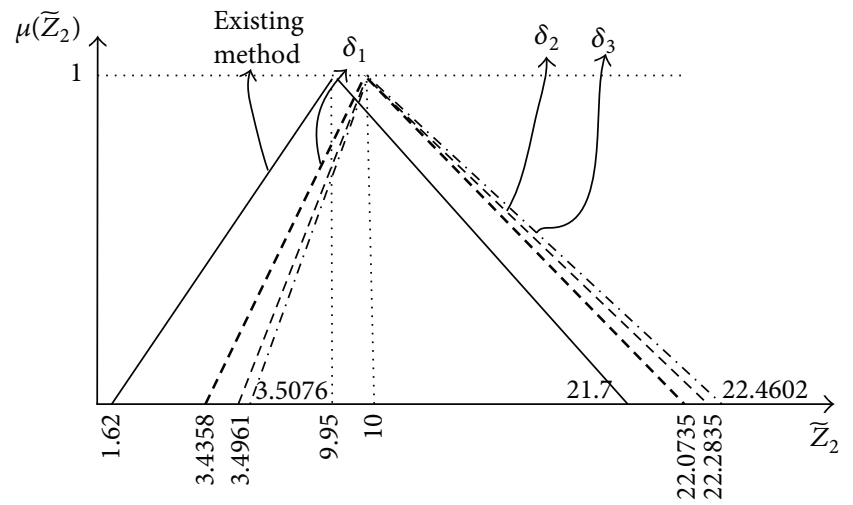

Figure 2: The membership function of the lower level fuzzy objective value provided by our approach and the existing method.

while there is a decrease of the upper bounds of the upper and lower level objective functions.

\section{Conclusion}

In this paper, a fully fuzzy bilevel linear programming problem in which all the coefficients and decision variables are fuzzy numbers is addressed. In order to solve such a problem, the concepts of the feasible region and the fuzzy optimal solution of the problem are first defined. Then the fully fuzzy bilevel linear programming can be converted into a deterministic bilevel programming problem by using the deviation degree measures and a ranking function method of fuzzy numbers. Taking into account the overall balance between improving the objective function value and decreasing the allowed deviation degree, a computational method to obtain a fuzzy optimal solution is presented. As one of the future works, the proposed method may be extended to handle fully fuzzy multilevel programming problems.

\section{Competing Interests}

The author declares that there is no conflict of interests regarding the publication of this paper.

\section{Acknowledgments}

This work was supported by Science Foundation of the Education Department of Shaanxi Province of China (no. 15JK1036) and Science Foundation of Baoji University of Arts and Sciences (no. ZK16049).

\section{References}

[1] J. F. Bard, Practical Bilevel Optimization: Algorithms and Applications, Kluwer Academic Publishers, Dordrecht, The Netherlands, 1998.

[2] S. Dempe, Foundations of Bilevel Programming, Kluwer Academic, Dordrecht, Netherlands, 2002.

[3] S. Dempe, V. Kalashnikov, G. A. Pérez-Valdés, and N. Kalashnykova, Bilevel Programming Problems: Theory, Algorithms and Applications to Energy Networks, Kluwer Academic, Dordrecht, Netherlands; Springer, Berlin, Germany, 2015.

[4] B. Colson, P. Marcotte, and G. Savard, "An overview of bilevel optimization," Annals of Operations Research, vol. 153, no. 1, pp. 235-256, 2007.

[5] B. Colson, P. Marcotte, and G. Savard, "Bilevel programming: a survey," 4OR, vol. 3, no. 2, pp. 87-107, 2005.

[6] S. Dempe, "Annotated bibliography on bilevel programming and mathematical programs with equilibrium constraints," Optimization, vol. 52, no. 3, pp. 333-359, 2003. 
[7] F. Gzara, "A cutting plane approach for bilevel hazardous material transport network design," Operations Research Letters, vol. 41, no. 1, pp. 40-46, 2013.

[8] P. Fontaine and S. Minner, "Benders decomposition for discrete-continuous linear bilevel problems with application to traffic network design," Transportation Research Part B: Methodological, vol. 70, pp. 163-172, 2014.

[9] M. Cecchini, J. Ecker, M. Kupferschmid, and R. Leitch, "Solving nonlinear principal-agent problems using bilevel programming," European Journal of Operational Research, vol. 230, no. 2, pp. 364-373, 2013.

[10] M. Labbé and A. Violin, "Bilevel programming and price setting problems," 4OR, vol. 11, no. 1, pp. 1-30, 2013.

[11] G. Zhang, G. Zhang, Y. Gao, and J. Lu, "Competitive strategic bidding optimization in electricity markets using bilevel programming and swarm technique," IEEE Transactions on Industrial Electronics, vol. 58, no. 6, pp. 2138-2146, 2011.

[12] V. V. Kalashnikov, S. Dempe, G. A. Pérez-Valdés, N. I. Kalashnykova, and J.-F. Camacho-Vallejo, "Bilevel programming and applications," Mathematical Problems in Engineering, vol. 2015, Article ID 310301, 16 pages, 2015.

[13] L. A. Zadeh, "Fuzzy sets," Information and Computation, vol. 8, no. 3, pp. 338-353, 1965.

[14] M. Sakawa, I. Nishizakia, and Y. Uemura, "Interactive fuzzy programming for multi-level linear programming problems with fuzzy parameters," Fuzzy Sets and Systems, vol. 109, no. 1, pp. 3-19, 2000.

[15] G. Q. Zhang and J. Lu, "The definition of optimal solution and an extended Kuhn-Tucker approach for fuzzy linear bilevel programming," IEEE Intelligent Informatics Bulletin, vol. 6, no. 2, pp. 1-7, 2005.

[16] G. Zhang and J. Lu, "Model and approach of fuzzy bilevel decision making for logistics planning problem," Journal of Enterprise Information Management, vol. 20, no. 2, pp. 178-197, 2007.

[17] G. Zhang, J. Lu, and T. Dillon, "Fuzzy linear bilevel optimization: solution concepts, approaches and applications," in Fuzzy Logic, vol. 215 of Studies in Fuzziness and Soft Computing, pp. 351-379, Springer, Berlin, Germany, 2007.

[18] S. Dempe and T. Starostina, On the Solution of Fuzzy Bilevel Programming Problems, Optimization Online, Freiberg, Germany, 2007.

[19] G. Q. Zhang, J. Lu, and T. Dillon, "Decentralized multi-objective bilevel decision making with fuzzy demands," Knowledge-Based Systems, vol. 20, no. 5, pp. 495-507, 2007.

[20] G. Q. Zhang, J. Lu, and Y. Gao, "Fuzzy bilevel programming: multi-objective and multi-follower with shared variables," International Journal of Uncertainty, Fuzziness and Knowledge-Based Systems, vol. 16, pp. 105-133, 2008.

[21] Y. Gao, G. Q. Zhang, and J. Lu, "A fuzzy multi-objective bilevel decision support system," International Journal of Information Technology and Decision Making, vol. 8, no. 1, pp. 93-108, 2009.

[22] G. Q. Zhang and J. Lu, "Fuzzy bilevel programming with multiple objectives and cooperative multiple followers," Journal of Global Optimization, vol. 47, no. 3, pp. 403-419, 2010.

[23] J. J. Buckley and T. Feuring, "Evolutionary algorithm solution to fuzzy problems: fuzzy linear programming," Fuzzy Sets and Systems, vol. 109, no. 1, pp. 35-53, 2000.

[24] F. H. Lotfi, T. Allahviranloo, M. A. Jondabeh, and L. Alizadeh, "Solving a full fuzzy linear programming using lexicography method and fuzzy approximate solution," Applied Mathematical Modelling, vol. 33, no. 7, pp. 3151-3156, 2009.
[25] A. Kumar, J. Kaur, and P. Singh, "A new method for solving fully fuzzy linear programming problems," Applied Mathematical Modelling. Simulation and Computation for Engineering and Environmental Systems, vol. 35, no. 2, pp. 817-823, 2011.

[26] H. Saberi Najafi and S. A. Edalatpanah, "A note on 'A new method for solving fully fuzzy linear programming problems,' Applied Mathematical Modelling, vol. 37, no. 14-15, pp. 78657867, 2013.

[27] Y. R. Fan, G. H. Huang, and A. L. Yang, "Generalized fuzzy linear programming for decision making under uncertainty: feasibility of fuzzy solutions and solving approach," Information Sciences, vol. 241, pp. 12-27, 2013.

[28] N. Safaei and M. Saraj, "A new method for solving fully fuzzy linear bilevel programming problems," International Journal of Applied Operational Research, vol. 4, no. 1, pp. 51-58, 2014.

[29] D. J. Dubois and H. Prade, Fuzzy Sets and Systems: Theory and applications, Academic Press, New York, NY, USA, 1980.

[30] T.-S. Liou and M. J. Wang, "Ranking fuzzy numbers with integral value," Fuzzy Sets and Systems, vol. 50, no. 3, pp. 247$255,1992$.

[31] H.-S. Lee, "Optimal consensus of fuzzy opinions under group decision making environment," Fuzzy Sets and Systems, vol. 132, no. 3, pp. 303-315, 2002.

[32] H. F. Cheng, W. L. Huang, Q. Zhou, and J. H. Cai, "Solving fuzzy multi-objective linear programming problems using deviation degree measures and weighted max-min method," Applied Mathematical Modelling, vol. 37, no. 10-11, pp. 6855-6869, 2013.

[33] S. M. Hashemi, M. Modarres, E. Nasrabadi, and M. M. Nasrabadi, "Fully fuzzified linear programming, solution and duality," Journal of Intelligent and Fuzzy Systems, vol. 17, no. 3, pp. 253-261, 2006. 


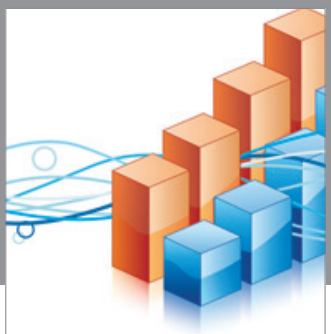

Advances in

Operations Research

vatem alat4

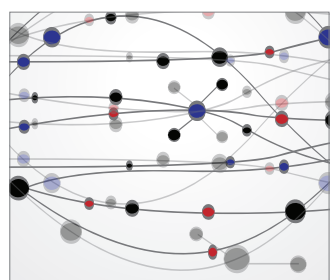

\section{The Scientific} World Journal
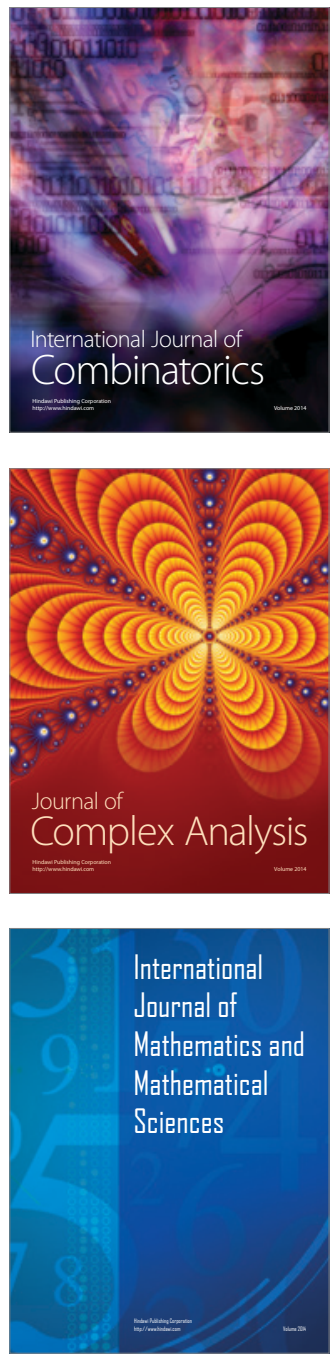
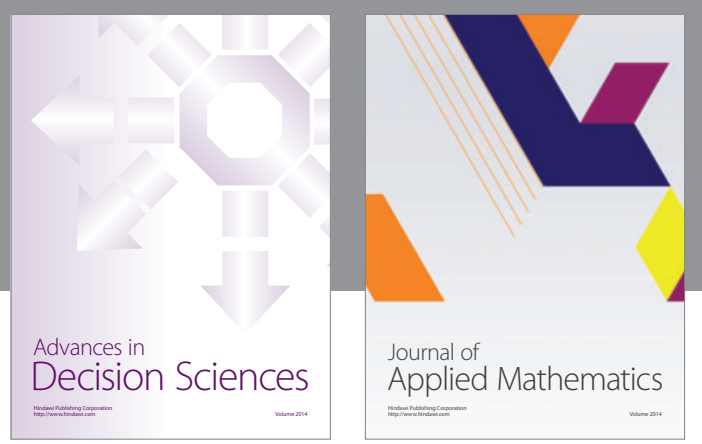

Algebra

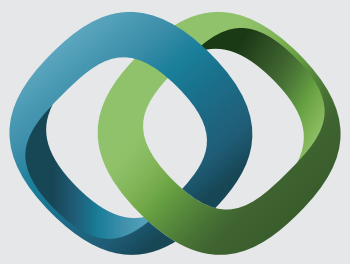

\section{Hindawi}

Submit your manuscripts at

http://www.hindawi.com
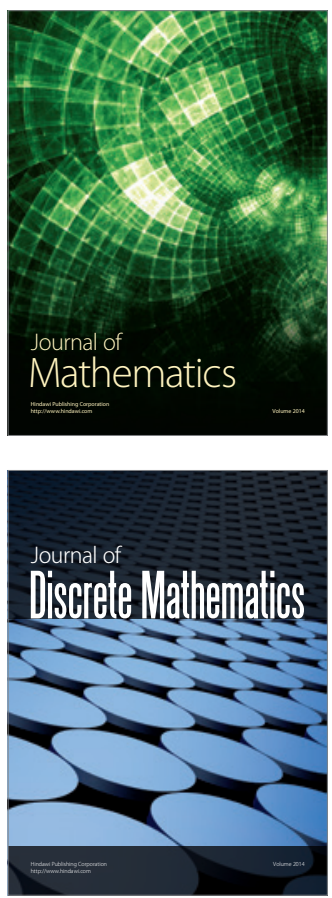

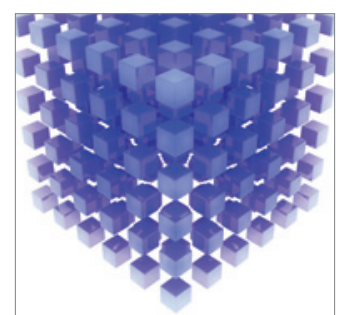

Mathematical Problems in Engineering
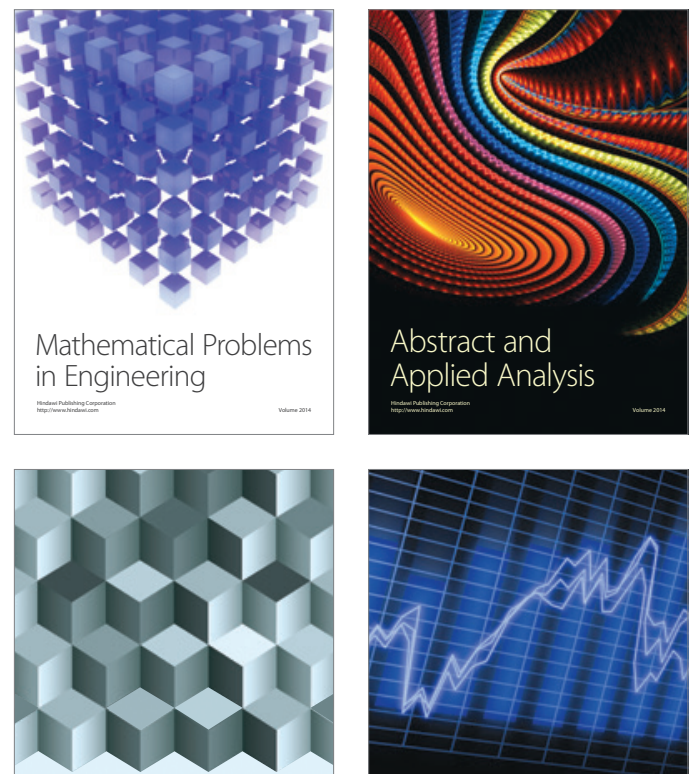

Journal of

Function Spaces

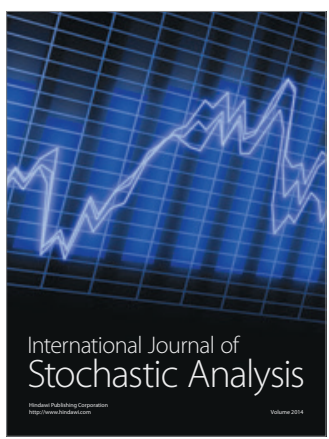

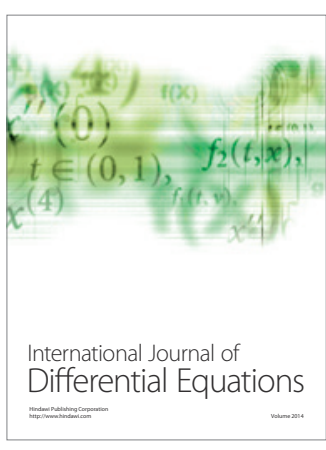
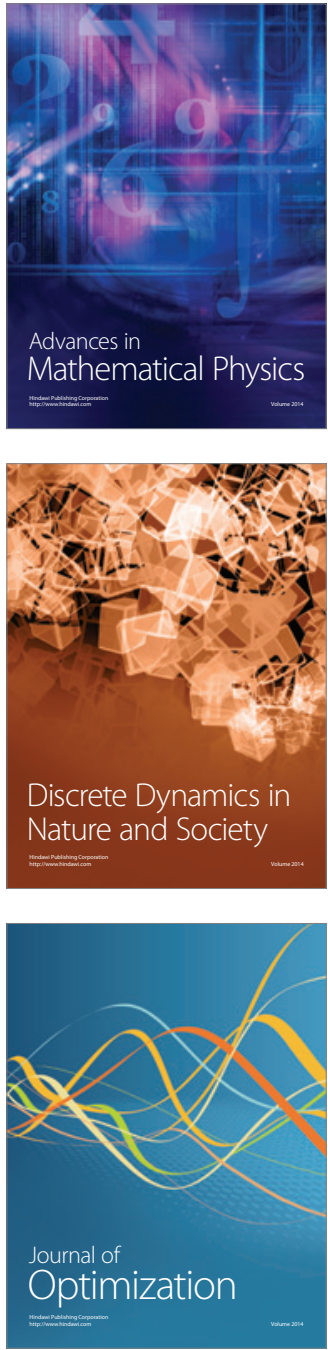